University of Texas at El Paso

ScholarWorks@UTEP

$3-2014$

\title{
Fuzzy Logic Ideas Can Help in Explaining Kahneman and Tversky's Empirical Decision Weights
}

Joe Lorkowski

The University of Texas at El Paso, lorkowski@computer.org

Vladik Kreinovich

The University of Texas at El Paso, vladik@utep.edu

Follow this and additional works at: https://scholarworks.utep.edu/cs_techrep

Part of the Computer Sciences Commons

Comments:

Technical Report: UTEP-CS-14-27

To appear in Proceedings of the 4th World Conference on Soft Computing, Berkeley, California, May 25-27, 2014.

\section{Recommended Citation}

Lorkowski, Joe and Kreinovich, Vladik, "Fuzzy Logic Ideas Can Help in Explaining Kahneman and Tversky's Empirical Decision Weights" (2014). Departmental Technical Reports (CS). 835.

https://scholarworks.utep.edu/cs_techrep/835

This Article is brought to you for free and open access by the Computer Science at ScholarWorks@UTEP. It has been accepted for inclusion in Departmental Technical Reports (CS) by an authorized administrator of ScholarWorks@UTEP. For more information, please contact Iweber@utep.edu. 


\title{
Fuzzy Logic Ideas Can Help in Explaining Kahneman and Tversky's Empirical Decision Weights
}

\author{
Joe Lorkowski and Vladik Kreinovich \\ Department of Computer Science \\ University of Texas at El Paso \\ El Paso, Texas 79968, USA \\ lorkowski@computer.org,vladik@utep.edu
}

\begin{abstract}
Analyzing how people actually make decisions, the Nobelist Daniel Kahneman and his co-author Amos Tversky found out that instead of maximizing the expected gain, people maximize a weighted gain, with weights determined by the corresponding probabilities. The corresponding empirical weights can be explained qualitatively, but quantitatively, these weights remains largely unexplained. In this paper, we show that with a surprisingly high accuracy, these weights can be explained by fuzzy logic ideas.
\end{abstract}

\section{EMPIRICAL DECISION WEIGHTS: FORMULATION OF THE PROBLEM}

Decisions are important. One of the main objectives of science and engineering is to help people make decisions. For example, we try to predict weather, so that people will be able to dress properly (and take an umbrella if needed), so that if a hurricane is coming, people can evacuate. We analyze quantum effects in semi-conductors so that engineers can design better computer chips. We analyze diseases so that medical doctors can help select the best treatment, etc.

In complex situations, people need help in making their decisions. In simple situations, an average person can easily make a decision. For example, if the weather forecast predicts rain, one should take an umbrella with him/her, otherwise one should not.

In more complex situations, however, even when we know all the possible consequences of each action, it is not easy to make a decision. For example, in medicine, many treatments come with side effects: a surgery can sometimes result in a patient's death, immune system suppression can result in a infectious disease, etc. In such situations, it is not easy to compare different actions, and even skilled experts would appreciate computer-based help.

To help people make decisions, we need to analyze how people make decisions. One of the difficulties in designing computer-based systems which would help people make decisions is that to make such systems successful, we need to know what exactly people want when they make decisions. Often, people cannot explain in precise terms why exactly they have selected this or that alternative.

In such situations, we need to analyze how people actually make decisions, and then try to come up with formal descriptions which fit the observed behavior.

Experiments start with decision making under full information. To analyze how people make decisions, researchers start with the simplest situations, in which we have the full information about the :

- we know all possible outcomes $o_{1}, \ldots, o_{n}$ of all possible actions;

- $\quad$ we know the exact value $u_{i}$ (e.g., monetary) of each outcome $o_{i}$; and

- for each action $a$ and to each outcome $i$, we know the probability $p_{i}(a)$ of this outcome.

Seemingly reasonable behavior. The outcome of each action $a$ is not deterministic. For the same action, we may get different outcomes $u_{i}$ with different probabilities $p_{i}(a)$. However, usually similar situations are repeated again and again.

If we repeat a similar situation several times, then the average expected gain of selecting an action $a$ becomes close to the mathematical expectation of the gain, i.e., to the value

$$
u(a) \stackrel{\text { def }}{=} \sum_{i=1}^{n} p_{i}(a) \cdot u_{i} .
$$

Thus, we expect that a decision maker selects the action $a$ for which this expected value $u(a)$ is the largest. In the first crude approximation, this is how people actually make decisions. But if we want a more precise description of human behavior, we - somewhat surprisingly - have to modify this formula.

How people actually make decisions is somewhat different. In their famous experiments, the Nobelist Daniel Kahneman and his co-author Amos Tversky found out that a much more accurate description of human decision making can be obtained if we assume that, instead of maximizing the expected gain, people maximize a weighted gain, with weights determined by the corresponding probabilities; see, e.g., [1] and references therein. 
In other words, people select the action $a$ for which the weighted gain

$$
w(a) \stackrel{\text { def }}{=} \sum_{i} w_{i}(a) \cdot u_{i}
$$

attains the largest possible value, where $w_{i}(a)=f\left(p_{i}(a)\right)$ for an appropriate function $f(x)$.

This empirical transformation $f(x)$ from probabilities to weights takes the following form:

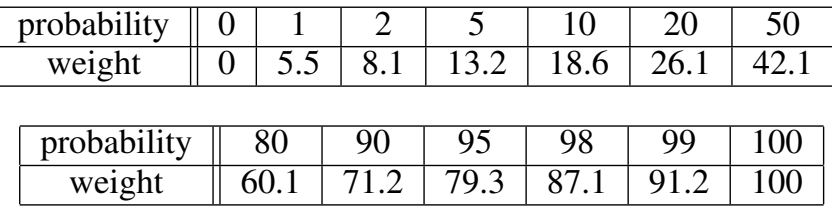

How can we explain this empirical transformation? There are qualitative explanations for this phenomenon, but not the quantitative one.

In this paper, we propose a quantitative explanation based on the fuzzy logic ideas.

\section{FUZZY-MOTIVATED IDEA: CONSIDERING "DistinguishaBLE" PROBABILITIES}

Main idea. The main idea behind our explanation is based on the fact that when people make decisions, they do not estimate probabilities as numbers from the interval $[0,1]$ and do not process them. If a person is asked about the probability of a certain event, in many cases, the answer will not come as an exact number, it will most probably come as an imprecise ("fuzzy") word, like "low", "high", "medium", etc.; see, e.g., [2], [4], [6].

In other words, instead of using all infinitely many possible real numbers from the interval $[0,1]$, we only use finitely many possible values - i.e., in effect, we estimate the probability on a finite scale. The reason for this discretization is that if the two probability values are close to each other, intuitively, we do not feel the difference. For example, there is a clear different between $10 \%$ chances of rain or $50 \%$ chances of rain, but we do not think that anyone can feel the difference between $50 \%$ and $51 \%$ chances. So, the discrete scale is formed by probabilities which are distinguishable from each other. Let us show how this idea can be formalized.

Comment. In this formalization, we will follow ideas first outlined in [3].

How to formalize when probabilities are distinguishable. Probability of an event is estimated, from observations, as the frequency with which this event occurs. For example, if out of 100 days of observation, rain occurred in 40 of these days, then we estimate the probability of rain as $40 \%$. In general, if out of $n$ observations, the event was observed in $m$ of them, we estimate the probability as the ratio

$$
\frac{m}{n} \text {. }
$$

This ratio is, in general, different from the actual (unknown) probability. For example, if we take a fair coin, for which the probability of head is exactly $50 \%$, and flip it 100 times, we may get 50 heads, but we may also get 47 heads, 52 heads, etc.

It is known (see, e.g., [5]), that the expected value of the frequency is equal to $p$, and that the standard deviation of this frequency is equal to

$$
\sigma=\sqrt{\frac{p \cdot(1-p)}{n}} .
$$

It is also known that, due to the Central Limit Theorem, for large $n$, the distribution of frequency is very close to the normal distribution (with the corresponding mean $p$ and standard deviation $\sigma$ ).

For normal distribution, we know that with a high certainty all the values are located within 2-3 standard deviations from the mean, i.e., in our case, within the interval

$$
\left(p-k_{0} \cdot \sigma, p+k_{0} \cdot \sigma\right),
$$

where $k_{0}=2$ or $k_{0}=3$ : for example, for $k_{0}=3$, this is true with confidence $99.9 \%$. We can thus say that the two values of probability $p$ and $p^{\prime}$ are (definitely) distinguishable if the corresponding intervals of possible values of frequency do not intersect - and thus, we can distinguish between these two probabilities just by observing the corresponding frequencies.

In precise terms, the probabilities $p<p^{\prime}$ are distinguishable if

$$
\left(p-k_{0} \cdot \sigma, p+k_{0} \cdot \sigma\right) \cap\left(p^{\prime}-k_{0} \cdot \sigma^{\prime}, p+k_{0} \cdot \sigma^{\prime}\right)=\emptyset,
$$

where

$$
\sigma^{\prime} \stackrel{\text { def }}{=} \sqrt{\frac{p^{\prime} \cdot\left(1-p^{\prime}\right)}{n}},
$$

i.e., if $p^{\prime}-k_{0} \cdot \sigma^{\prime} \geq p+k_{0} \cdot \sigma$. The smaller $p^{\prime}$, the smaller the difference $p^{\prime}-k_{0} \cdot \sigma$. Thus, for a given probability $p$, the next distinguishable value $p^{\prime}$ is the one for which

$$
p^{\prime}-k_{0} \cdot \sigma^{\prime}=p+k_{0} \cdot \sigma \text {. }
$$

When $n$ is large, these value $p$ and $p^{\prime}$ are close to each other; therefore, $\sigma^{\prime} \approx \sigma$. Substituting an approximate value $\sigma$ instead of $\sigma^{\prime}$ into the above equality, we conclude that

$$
p^{\prime} \approx p+2 k_{0} \cdot \sigma=p+2 k_{0} \cdot \frac{p \cdot(1-p)}{n} .
$$

If the value $p$ corresponds to the $i$-th level out of $m$-i.e., in fuzzy terms, corresponds to the truth value

$$
\frac{i}{m}
$$

- then the next value $p^{\prime}$ corresponds to the $(i+1)$-st level, i.e., to the truth value

$$
\frac{i+1}{m} \text {. }
$$

Let $g(p)$ denote the fuzzy truth value corresponding to the probability $p$. Then,

$$
g(p)=\frac{i}{m} \text { and } g\left(p^{\prime}\right)=\frac{i+1}{m} .
$$

Since the values $p$ and $p^{\prime}$ are close, the difference $p^{\prime}-p$ is small, and therefore, we can expand the expression $g\left(p^{\prime}\right)=$ 
$g\left(p+\left(p^{\prime}-p\right)\right)$ in Taylor series and keep only linear terms in this expansion: $g\left(p^{\prime}\right) \approx g(p)+\left(p^{\prime}-p\right) \cdot g^{\prime}(p)$, where

$$
g^{\prime}(p)=\frac{d g}{d p}
$$

denotes the derivative of the function $g(p)$. Thus,

$$
g\left(p^{\prime}\right)-g(p)=\frac{1}{m}=\left(p^{\prime}-p\right) \cdot g^{\prime}(p) .
$$

Substituting the known expression for $p^{\prime}-p$ into this formula, we conclude that

$$
\frac{1}{m}=2 k_{0} \cdot \sqrt{\frac{p \cdot(1-p)}{n}} \cdot g^{\prime}(p) .
$$

This can be rewritten as

$$
g^{\prime}(p) \cdot \sqrt{p \cdot(1-p)}=\text { const }
$$

for some constant, and thus,

$$
g^{\prime}(p)=\text { const } \cdot \frac{1}{\sqrt{p \cdot(1-p)}} .
$$

Integrating this expression and taking into account that $p=0$ corresponds to the lowest 0 -th level - i.e., that $g(0)=0$ - we conclude that

$$
g(p)=\text { const } \cdot \int_{0}^{p} \frac{d q}{\sqrt{q \cdot(1-q)}} .
$$

This integral can be easily computed if introduce a new variable $t$ for which $q=\sin ^{2}(t)$. In this case,

$$
d q=2 \cdot \sin (t) \cdot \cos (t) \cdot d t,
$$

$1-p=1-\sin ^{2}(t)=\cos ^{2}(t)$ and therefore,

$$
\sqrt{p \cdot(1-p)}=\sqrt{\sin ^{2}(t) \cdot \cos ^{2}(t)}=\sin (t) \cdot \cos (t) .
$$

The lower bound $q=0$ corresponds to $t=0$ and the upper bound $q=p$ corresponds to the value $t_{0}$ for which $\sin ^{2}\left(t_{0}\right)=$ $p-$ i.e., $\sin \left(t_{0}\right)=\sqrt{p}$ and $t_{0}=\arcsin (\sqrt{p})$. Therefore,

$$
\begin{gathered}
g(p)=\text { const } \cdot \int_{0}^{p} \frac{d q}{\sqrt{q \cdot(1-q)}}= \\
\text { const } \cdot \int_{0}^{t_{0}} \frac{2 \cdot \sin (t) \cdot \cos (t) \cdot d t}{\sin (t) \cdot \cos (t)}=\int_{0}^{t_{0}} 2 \cdot d t= \\
2 \cdot \text { const } \cdot t_{0} .
\end{gathered}
$$

We know how $t_{0}$ depends on $p$, so we get

$$
g(p)=2 \cdot \operatorname{const} \cdot \arcsin (\sqrt{p}) .
$$

We can determine the constant from the condition that the largest possible probability value $p=1$ should correspond to the right-most point $g(p)=1$. From the condition that $g(1)=1$, taking into account that

$$
\arcsin (\sqrt{1})=\arcsin (1)=\frac{\pi}{2}
$$

we conclude that

$$
g(p)=\frac{2}{\pi} \cdot \arcsin (\sqrt{p}) .
$$

Description of the resulting discretization. For a scale from 0 to some number $m$, the value $g(m)$ is equal to the ratio

$$
\frac{i}{m} \text {. }
$$

So, $i=m \dot{g}(p)$.

Thus, the desired discretization means that to each probability $p$, we assign the value $i \approx m \cdot g(p)$ on the scale from 0 to $m$, where $g(p)$ is described by the above formula.

\section{Distinguishable Probabilities Can ExPlain EMPIRICAL DECISION WEIGHTS}

How do we select weights? If we need to select finitely many weights from the interval $[0,1]$, then it is natural to select weights which are equally distributed on this interval, i.e., weights

$$
0, \frac{1}{m}, \frac{2}{m}, \ldots, \frac{m-1}{m}, 1 \text {. }
$$

How to assign weights to probabilities: idea. We have $m$ a finite list of distinguishable probabilities $0=p_{0}<p_{1}<\ldots<$ $p_{m}=1$. These probabilities correspond to degree

$$
g\left(p_{i}\right)=\frac{i}{m}
$$

where $g(p)$ is determined by the formula (1). We need to assign, to each of these probabilities, an appropriate weight from the above list (2).

The larger the probability, the more weight we should assign to the corresponding outcome. Thus, we arrive at the following assignment of weights to probabilities:

- $\quad$ to the value $p_{0}=0$, we assign the smallest possible weight $w_{0}=0$;

- $\quad$ to the next value $p_{1}$, we assign the next weight

$$
w_{1}=\frac{1}{m}
$$

- $\quad$ to the next value $p_{2}$, we assign the next weight

$$
w_{1}=\frac{1}{m}
$$

- $\quad \ldots$

- to the value $p_{m-1}$, we assign the weight

$$
w_{m-1}=\frac{m-1}{m}
$$

- finally, to the value $p_{m}=1$, we assign the weight

$$
w_{m}=1 \text {. }
$$

In general, to the value $p_{i}$, we assign the weight

$$
w_{i}=\frac{i}{m} \text {. }
$$

By comparing this assignment with the formula (3), we conclude that to each value $p_{i}$, we assign the value $w_{i}=g\left(p_{i}\right)$. 
How to assign weights to probabilities: result. Our arguments show that to each probability $p \in[0,1]$, we assign the weight $g(p)$, where the function $g(p)$ is determined by the formula (1).

Comparing our weights with empirical weights: first try. Let us compare the probabilities $p_{i}$, Kahneman's empirical weights $\widetilde{w}_{i}$, and the weight $w_{i}=g\left(p_{i}\right)$ computed by using the formula (1):

\begin{tabular}{|c||c|c|c|c|c|c|c|}
\hline$p_{i}$ & 0 & 1 & 2 & 5 & 10 & 20 & 50 \\
\hline$\widetilde{w}_{i}$ & 0 & 5.5 & 8.1 & 13.2 & 18.6 & 26.1 & 42.1 \\
\hline$w_{i}=g\left(p_{i}\right)$ & 0 & 6.4 & 9.0 & 14.4 & 20.5 & 29.5 & 50.0 \\
\hline
\end{tabular}

\begin{tabular}{|c||c|c|c|c|c|c|}
\hline$p_{i}$ & 80 & 90 & 95 & 98 & 99 & 100 \\
\hline$\widetilde{w}_{i}$ & 60.1 & 71.2 & 79.3 & 87.1 & 91.2 & 100 \\
\hline$w_{i}=g\left(p_{i}\right)$ & 70.5 & 79.5 & 85.6 & 91.0 & 93.6 & 100 \\
\hline
\end{tabular}

The estimates $w_{i}=g\left(p_{i}\right)$ are closer to the observed weights $\widetilde{w}_{i}$ than the original probabilities, but the relation does not seem very impressive.

We will show that the fit is much better than it seems at first glance. At first glance, the above direct comparison between the observed weights $\widetilde{w}_{i}$ and the estimated weights $w_{i}=g\left(p_{i}\right)$ seems to make perfect sense. However, let us look deeper.

The weights come from the fact that users maximize the weighted gain $w(a)=\sum w_{i}(a) \cdot u_{i}$. It is easy to observe that if we multiply all the weights by the same positive constant $\lambda>0$, i.e., consider the weights $w_{i}^{\prime}(a)=\lambda \cdot w_{i}(a)$, then for each action, the resulting value of the weighted gain will also increase by the same factor:

$$
\begin{gathered}
w^{\prime}(a)=\sum w_{i}^{\prime}(a) \cdot u_{i}=\sum \lambda \cdot w_{i}(a) \cdot u_{i}= \\
\lambda \cdot \sum w_{i}(a) \cdot u_{i}=\lambda \cdot w_{i}(a) .
\end{gathered}
$$

The relation between the weighted gains of two actions $a$ and $a^{\prime}$ does not change if we simply multiply both gains by a positive constant:

- $\quad$ if $w_{i}(a)<w_{i}\left(a^{\prime}\right)$, then, multiplying both sides of this inequality by $\lambda$, we get $w_{i}^{\prime}(a)<w_{i}^{\prime}\left(a^{\prime}\right)$;

- $\quad$ if $w_{i}(a)=w_{i}\left(a^{\prime}\right)$, then, multiplying both sides of this equality by $\lambda$, we get $w_{i}^{\prime}(a)=w_{i}^{\prime}\left(a^{\prime}\right)$;

- $\quad$ if $w_{i}(a)>w_{i}\left(a^{\prime}\right)$, then, multiplying both sides of this inequality by $\lambda$, we get $w_{i}^{\prime}(a)>w_{i}^{\prime}\left(a^{\prime}\right)$.

All we observe if which of the two actions a person selects. Since multiplying all the weights by a constant does not change the selection, this means that based on the selection, we cannot uniquely determine the weights: an empirical selection which is consistent with the weights $w_{i}$ is equally consistent with the weights $w_{i}^{\prime}=\lambda \cdot w_{i}$.

This fact can be use to normalize the empirical weights, i.e., to multiply them by a constant so as to satisfy some additional condition.
In [1], to normalize the weights, the authors use the requirement that the weight corresponding to probability 1 should be equal to 1 . Since for $p=1$, the estimated weight $g(1)$ is also equal to 1 , we get a perfect match for $p=1-$ but a rather lousy march for probabilities intermediate between 0 and 1 .

Instead of this normalization, we can select $\lambda$ so as to get the best match "on average".

How to improve the fit: details. A natural idea is to select $\lambda$ from the Least Squares method, i.e., select $\lambda$ for which the relative mean squares difference

$$
\sum_{i}\left(\frac{\lambda \cdot w_{i}-\widetilde{w}_{i}}{w_{i}}\right)^{2}
$$

is the smallest possible. Differentiating this expression with respect to $\lambda$ and equating the derivative to 0 , we conclude that

$$
\sum_{i}\left(\lambda-\frac{\widetilde{w}_{i}}{w_{i}}\right)=0
$$

i.e., that

$$
\lambda=\frac{1}{m} \cdot \sum_{i} \frac{\widetilde{w}_{i}}{w_{i}}
$$

Resulting match. For the above values, this formula leads to $\lambda=0.910$.

The resulting values $w_{i}^{\prime}=\lambda \cdot w_{i}$ are indeed much closer to the empirical weights $\widetilde{x}_{i}$ :

\begin{tabular}{|c||c|c|c|c|c|c|c|}
\hline$p_{i}$ & 0 & 1 & 2 & 5 & 10 & 20 & 50 \\
\hline$\widetilde{w}_{i}$ & 0 & 5.5 & 8.1 & 13.2 & 18.6 & 26.1 & 42.1 \\
\hline$w_{i}^{\prime}=\lambda \cdot g\left(p_{i}\right)$ & 0 & 5.8 & 8.2 & 13.1 & 18.7 & 26.8 & 45.5 \\
\hline
\end{tabular}

\begin{tabular}{|c||c|c|c|c|c|c|}
\hline$p_{i}$ & 80 & 90 & 95 & 98 & 99 & 100 \\
\hline$\widetilde{w}_{i}$ & 60.1 & 71.2 & 79.3 & 87.1 & 91.2 & 100 \\
\hline$w_{i}^{\prime}=\lambda \cdot g\left(p_{i}\right)$ & 64.2 & 72.3 & 77.9 & 82.8 & 87.4 & 91.0 \\
\hline
\end{tabular}

For most probabilities $p_{i}$, the difference between the fuzzymotivated weights $w_{i}^{\prime}$ and the empirical weights $\widetilde{w}_{i}$ is so small that it is below the accuracy with which the empirical weights can be obtained from the experiment.

Conclusion. Fuzzy-motivated ideas indeed explain Kahneman and Tversky's empirical decision weights.

\section{ACKNOWLEDGMENT}

This work was supported in part by the National Science Foundation grants HRD-0734825 and HRD-1242122 (CyberShARE Center of Excellence) and DUE-0926721. 


\section{REFERENCES}

[1] D. Kahneman, Thinking, Fast and Slow, Farrar, Straus, and Giroux, New York, 2011.

[2] G. Klir and B. Yuan, Fuzzy Sets and Fuzzy Logic, Prentice Hall, Upper Saddle River, New Jersey, 1995.

[3] H. T. Nguyen, V. Kreinovich, and B. Lea, "How to combine probabilistic and fuzzy uncertainties in fuzzy control", Proceedings of the Second International Workshop on Industrial Applications of Fuzzy Control and Intelligent Systems, College Station, December 2-4, 1992, pp. 117-121.
[4] H. T. Nguyen and E. A. Walker, A First Course in Fuzzy Logic, Chapman and Hall/CRC, Boca Raton, Florida, 2006.

[5] D. J. Sheskin, Handbook of Parametric and Nonparametric Statistical Procedures, Chapman \& Hall/CRC, Boca Raton, Florida, 2011.

[6] L. A. Zadeh, "Fuzzy sets", Information and Control, 1965, Vol. 8, pp. 338-353. 